\title{
IMPROVED SMOOTHED PARTICLE HYDRODYNAMICS WITH RANS FOR FREE-SURFACE FLOW PROBLEMS
}

\author{
J. R. SHAO*, M. B. $\mathrm{LIU}^{\dagger}$ and X. F. YANG ${ }^{\ddagger}$ \\ Institute of Mechanics \\ Chinese Academy of Sciences \\ No. 15 Beisihuanxi Road, Beijing, P. R. China \\ *shaojiaru@imech.ac.cn \\ †liumoubin@imech.ac.cn \\ ¥kanty@126.com \\ L. CHENG \\ Sichuan University \\ No. 24 South Section 1, Yihuan Road, Chengdu, China \\ xo-chenglin@163.com \\ Received 18 October 2010 \\ Accepted 2 May 2011
}

\begin{abstract}
This paper presents an implementation of an improved smoothed particle hydrodynamics (SPH) method for numerical simulation of free-surface flow problems. The presented SPH method involves two major modifications on the traditional SPH method: (1) kernel gradient correction (KGC) and density correction to improve the computational accuracy in particle approximation and (2) RANS turbulence model to capture the inherent physics of flow turbulence. In the simulation, artificial compressibility for modeling incompressible fluid and ghost particles for treating solid boundaries are both applied. The presented SPH has been applied to two dam-breaking problems. We demonstrated that the presented SPH method has very good performance with more accurate flow patterns and pressure field distribution.
\end{abstract}

Keywords: SPH; kernel gradient correction; RANS; artificial compressibility.

\section{Introduction}

Free-surface flow motions, especially violent wave motions, are common phenomena in coastal hydrodynamics and offshore engineering. When the violent wave encounters obstacles, very large impact and pressure oscillating can occur together with wave breaking and overturning. This can destroy the structure of the nearby obstacles. If the violent waves occur in coast areas, they may cause potential damages of property and loss of lives. Therefore, studying the flow mechanism of

\footnotetext{
$\dagger$ Corresponding author.
} 
the free-surface flows is of great significance in theory and practice [Mei (1989); Peregrine (2003); Faltinsen et al. (2004)].

In the past several decades, many scholars have already conducted some experiments [Buchner 2002; Jánosi et al. (2004)] and numerical simulations about free-surface problem. However, if the conditions are complex, the experiments will be expensive, or even cannot be implemented. Numerical simulation has become a great tool to predict fluid in coast hydrodynamics and offshore engineering.

Conventional grid-based numerical methods such as finite difference method (FDM) and finite volume method (FVM) have been widely applied to various areas of computational fluid dynamics (CFD) and currently are the dominant methods in numerical simulations of domain discretization and numerical discretization. In addition, many numerical techniques have been proposed to track the free surface, such as the volume of fluid (VOF) method [Hirt and Nichols (1981)], the Level-Set method [Sethian (2003)] and the CIP-based method [Hu and Kashiwagi (2004)]. Despite the great success, these numerical methods suffer from inherent difficulties in many aspects. Constructing a regular grid for irregular or complex geometry has never been an easy task, and usually requires additional complex mathematical transformation that can be even more expensive than solving the problem itself. Moreover, these methods have difficulties in avoiding numerical dissipation.

A recent strong interest has been focused on the development of the next generation computational methods, mesh-free method, such as molecular dynamics method (MD). [Berendsen et al. (1984)], dissipative particle dynamics method (DPD) [Groot and Warren (1997)], and smoothed particle hydrodynamics (SPH) [Gingold and Monaghan (1977); Lucy (1977); Monaghan (2005); Liu et al. (2008)]. $\mathrm{SPH}$ is a fully Lagrangian mesh-free technique, it is first introduced to resolve astrophysical problems in three-dimensional (3D) open space. In the SPH method, the state of a system is represented by a set of particles, which possess individual material properties and move according to the governing conservation equations. SPH features a remarkable flexibility in handling complex flow fields and including physical effects. The special advantage of the SPH is the adaptive nature; therefore, the formulation of SPH is not affected by the arbitrariness of the particles and it can naturally handle problems with extremely large deformation [Liu and Liu, (2003)].

Despite the great advantages, conventional SPH method also has some problems. The accuracy and numerical stability are not good enough, especially in the boundary and the domains with uniform particles distribution. Besides, the governing equations of conventional SPH cannot guarantee the conservation of the total angular momentum. To resolve these problems, many improved methods are presented to recover the compatibility of the kernel approximation [Bonet and Lok (1999); Dalrymple and Rogers (2006)], and some have good effects. However, they only pay attention to the kernel and overlook the roles of the kernel gradient. A finite particle method (FPM) [Liu et al. (2005)] is used to modified the kernel gradient. Similar to FPM, this paper implemented a simplified form that is using a matrix to modify 
the kernel gradient so as to guarantee the second-order accuracy in the whole flow field. This kernel gradient corrected method can improve the accuracy and stability of conventional SPH method obviously.

Another important issue concerning free-surface flow is the turbulences in the surf zone. Direct numerical simulation (DNS) [Moin and Mahesh (1998); Moser et al. (1999)] has very high accuracy; however, this method needs so many meshes or particles and it is unrealistic to simulate complex flow fields with large computational domain. Therefore, in order to describe the turbulence effects in the flow field, suitable turbulence models [Chen and Jaw (1998)] are necessary. In this paper, the Reynolds averaged Navier-Stokes (RANS) are used to simulate the turbulence effects in the flow field [Anderson and Wendt (1995); Catalano and Amato (2003)]. The N-S equations are averaged over the time, and a suitable eddy viscosity assumption is used to satisfy the condition of closure of the Reynolds equation. It is shown that this method can lead to good results with less computational time.

\section{Methodology}

SPH is a meshfree, Lagrangian particle method for modeling fluid flows. SPH was first invented to solve astrophysical problems in 3D open space. The SPH method has also been applied extensively to a vast range of problems in either computational fluid or solid mechanics because of relatively strong ability to incorporate complicated physical effects into the SPH formulations. SPH has been extended to dynamic response with material strength as well as dynamic fluid flows with large deformations.

In the SPH method, the state of a system is represented by a set of particles, which possess individual material properties and move according to the governing conservation equations. The particles represent the problem domain and to act as the computational frame for field variable approximations. The SPH approximation does not require a predefined mesh to provide any connective of the particles in the process of computation, and it work well even without any particle refinement operation. Besides, SPH can combine the Lagrangian formulation with particle approximation harmoniously.

\subsection{The PDE equations in conventional SPH}

The continuity and momentum equations for a Newtonian fluid flow in Lagrangian coordinate system are

$$
\begin{aligned}
& \frac{\mathrm{d} \rho}{\mathrm{d} t}=-\rho \nabla \bullet \boldsymbol{\nu}, \\
& \frac{\mathrm{d} v}{\mathrm{~d} t}=-\frac{1}{\rho} \nabla p+\frac{\mu}{\rho} \nabla^{2} \boldsymbol{\nu}+\boldsymbol{g},
\end{aligned}
$$

where, $\rho, \boldsymbol{\nu}, p, \boldsymbol{g}$, and $\mu$ denote density, velocity vector, pressure, gravity, and dynamic viscosity, respectively. 
In SPH method, the values of a particular quantity at any point can be obtained using the following interpolation:

$$
\begin{aligned}
\left\langle f\left(\boldsymbol{x}_{i}\right)\right\rangle & =\sum_{j=1}^{N} \frac{m_{j}}{\rho_{j}} f\left(\boldsymbol{x}_{j}\right) W\left(\boldsymbol{x}_{i}-\boldsymbol{x}_{j}, h\right), \\
\left\langle\nabla f\left(\boldsymbol{x}_{i}\right)\right\rangle & =\sum_{j=1}^{N} \frac{m_{j}}{\rho_{j}} f\left(\boldsymbol{x}_{j}\right) \nabla_{i} W_{i j},
\end{aligned}
$$

where, $\left\langle f\left(\boldsymbol{x}_{i}\right)\right\rangle$ is the approximate value of particle $i, f\left(\boldsymbol{x}_{j}\right)$ is the value of $f(\boldsymbol{x})$ associated with particle $j, \boldsymbol{x}_{i}$ and $\boldsymbol{x}_{j}$ are the positions of corresponding particles, $h$ is the smooth length, $N$ is the number of the particles in the support domain, and $W$ is the smoothing function and represents a weighted contribution of particle $j$ to particle $i$. Therefore, the SPH forms of N-S equations can be obtained. It is given by

$$
\begin{aligned}
\frac{d \rho_{i}}{\mathrm{~d} t} & =\sum_{j=1}^{N} m_{j} \boldsymbol{\nu}_{i j} \bullet \nabla_{i} W_{i j}, \\
\frac{\mathrm{d} \boldsymbol{\nu}_{i}}{\mathrm{~d} t} & =-\sum_{j=1}^{N} m_{j}\left(\frac{p_{i}}{\rho_{i}^{2}}+\frac{p_{j}}{\rho_{j}^{2}}\right) \nabla_{i} W_{i j}+\sum_{j=1}^{N} \frac{4 m_{j}\left(\mu_{i}+\mu_{j}\right) \boldsymbol{x}_{i j} \bullet \nabla_{i} W_{i j}}{\left(\rho_{i}+\rho_{j}\right)^{2}\left(x_{i j}^{2}+0.01 h^{2}\right)} \boldsymbol{\nu}_{i j}+\boldsymbol{g},
\end{aligned}
$$

\subsection{Kernel function in conventional SPH}

The kernel function (also called smoothing function) is an important part in SPH simulation. It not only determines the pattern for the function approximation and defines the dimension of the support domain of particles, but also determines the consistency and hence the accuracy of both the kernel and particle approximations.

In the simulation, we use the cubic spline [Monaghan (1985)], because this kernel has continuous second-order derivative, and it is not sensitive to particle disorder.

$$
W(R, h)=a_{d} \times \begin{cases}1-\frac{3}{2} R^{2}+\frac{3}{4} R^{3}, & 0 \leq R<1 \\ \frac{1}{4}(2-R)^{3}, & 1 \leq R<2, \\ 0, & R \geq 2\end{cases}
$$

where $a_{d}$ is $3 / 2 h, 10 / 7 \pi h^{2}$, and $1 / \pi h^{3}$ in $1 \mathrm{D}, 2 \mathrm{D}$, and $3 \mathrm{D}$ spaces, respectively, so that the condition of unity can be satisfied for all the three dimensions. $R$ is the relative distance between two particles at point $i$ and $j, R=\left|\boldsymbol{x}_{i}-\boldsymbol{x}_{j}\right| / h$. The support domain of this kernel has a radius of $2 h$, when $R>2$, the interactions are zero.

\subsection{Modeling incompressible flow with artificial compressibility}

In the SPH method, an artificial compressibility technique is used to model the incompressible flow as a slightly compressible flow. The artificial compressibility 
considers that every theoretically incompressible fluid is actually compressible. Therefore, it is feasible to use a quasi-incompressible equation of state to model the incompressible flow. The purpose of introducing the artificial compressibility is to produce the time derivative of pressure. In this work, the artificial equation of state is

$$
p=c^{2} \rho,
$$

where $c$ is the sound speed which is a key factor that deserves careful consideration. If the actual sound speed is employed, the real fluid is approximated as an artificial fluid, which is ideally incompressible. Monaghan argued that the relative density variation $\delta$ is related to the fluid bulk velocity and sound speed in the following way [Monaghan (1994)]:

$$
\delta=\frac{\Delta \rho}{\rho_{0}}=\frac{\left|\rho-\rho_{0}\right|}{\rho_{0}}=\frac{V_{b}^{2}}{c^{2}}=M^{2},
$$

where $\rho_{0}, \Delta \rho, V_{b}$, and $\mathrm{M}$ are the initial density, absolute density variation, fluid bulk velocity, and Mach number, respectively.

Morris, through considering the balance of pressure, viscous force, and body force, proposed an estimate for the sound speed [Morris et al. 1997]. He argued that the square of the sound speed should be comparable with the largest value of $V_{b}^{2} / \delta, v V_{b} / \delta l$, and $F l / \delta$, i.e.

$$
c^{2}=\max \left(\frac{V_{b}^{2}}{\delta}, \frac{v V_{b}}{\delta l}, \frac{F l}{\delta}\right),
$$

where $v(v=\mu / \rho)$ is the kinetic viscosity, $F$ is the magnitude of the external body force, and $l$ is the characteristic length scale.

\subsection{Artificial viscosity}

Artificial viscosity can diffuse sharp variations in the flow and dissipate the energy of high-frequency term. Here, Monaghan type artificial viscosity is added to physical pressure term.

$$
\begin{aligned}
\frac{\mathrm{d} \boldsymbol{\nu}_{i}}{\mathrm{~d} t} & =-\sum_{j=1}^{N} m_{j}\left(\frac{p_{i}}{\rho_{i}^{2}}+\frac{p_{j}}{\rho_{j}^{2}}+\Pi_{i j}\right) \bullet \nabla_{i} W_{i j}+\boldsymbol{F}, \\
\prod_{i j} & = \begin{cases}\frac{-\alpha_{\Pi} \overline{c_{i j}} \phi_{i j}}{\overline{\rho_{i j}}}, & \boldsymbol{\nu}_{i j} \bullet x_{i j}<0 \\
0, & \boldsymbol{\nu}_{i j} \bullet x_{i j}<0,\end{cases}
\end{aligned}
$$

where

$$
\begin{aligned}
\phi_{i j} & =\frac{h_{i j} v_{i j} x_{i j}}{\left|x_{i j}\right|^{2}+\varphi^{2}}, \quad \overline{c_{i j}}=\frac{1}{2}\left(c_{i}+c_{j}\right), \quad \overline{\rho_{i j}}=\frac{1}{2}\left(\rho_{i}+\rho_{j}\right), \\
h_{i j} & =\frac{1}{2}\left(h_{i}+h_{j}\right), \quad v_{i j}=\left(\boldsymbol{\nu}_{i}-\boldsymbol{\nu}_{j}\right), \quad x_{i j}=\left(\boldsymbol{x}_{i}-\boldsymbol{x}_{j}\right) .
\end{aligned}
$$




\subsection{KGC SPH model with density correction}

In the SPH simulation, the computational accuracy is sensitive to the distribution of the particles. In some domains, the consistency conditions are no longer satisfied exactly and the characters of kernel function will not be satisfied.

If the density is not suitable, through the state equation, strong pressure shocks will appear in the pressure field. Therefore, using the original kernel function cannot obtain the accurate results. Therefore, a density correction is necessary.

$$
\rho_{i}^{\text {new }}=\sum_{j=1}^{N} \rho_{j} W_{i j}^{\text {new }} \frac{m_{j}}{\rho_{j}}=\sum_{j=1}^{N} m_{j} W_{i j}^{\text {new }},
$$

Here, we use two methods to modify the density. The first is renormalizing the kernel function every 20 time steps. The new kernel function can be described as follows:

$$
W_{i j}^{\text {new }}=\frac{W_{i j}}{\sum_{j=1}^{N} W_{i j} \frac{m_{j}}{\rho_{j}}} .
$$

The second is Moving least square (MLS) method. Here,

$$
\begin{aligned}
& W_{i j}^{\mathrm{MLS}}=W_{j}^{\mathrm{MLS}}\left(\boldsymbol{r}_{i}\right)=\beta\left(\boldsymbol{r}_{i}\right) \bullet\left(\boldsymbol{r}_{i}-\boldsymbol{r}_{j}\right) W_{i j}, \\
& W_{i j}^{\mathrm{MLS}}=\left[\beta_{0}\left(\boldsymbol{r}_{i}\right)+\beta_{x}\left(\boldsymbol{r}_{i}\right)\left(x_{i}-x_{j}\right)+\beta_{y}\left(\boldsymbol{r}_{i}\right)\left(y_{i}-y_{j}\right)\right] W_{i j},
\end{aligned}
$$

where, $x_{i}, y_{i}$ and $x_{j}, y_{j}$ are the coordinates of particle $i$ and $j$ in $x$ and $y$ directions separately.

$$
\begin{aligned}
\beta\left(r_{i} \rightarrow\right) & =\left[\begin{array}{l}
\beta_{0} \\
\beta_{x} \\
\beta_{y}
\end{array}\right]=A^{-1}\left[\begin{array}{l}
1 \\
0 \\
0
\end{array}\right], \\
A & =\sum_{j=1}^{N} W_{j}\left(\boldsymbol{r}_{i}\right) A^{\prime} V_{j}, \\
A^{\prime} & =\left[\begin{array}{ccc}
1 & x_{i}-x_{j} & y_{i}-y_{j} \\
x_{i}-x_{j} & \left(x_{i}-x_{j}\right)^{2} & \left(x_{i}-x_{j}\right)\left(y_{i}-y_{j}\right) \\
\left(y_{i}-y_{j}\right) & \left(x_{i}-x_{j}\right)\left(y_{i}-y_{j}\right) & \left(y_{i}-y_{j}\right)^{2}
\end{array}\right],
\end{aligned}
$$

Equation (4) shows that the kernel gradient has a significant influence on the accuracy. Considering the interaction between particles, a new kernel gradient model is used in the simulation. Based on Taylor series expansion on the SPH approximation of a function,

$$
\begin{aligned}
\int_{\Omega} f\left(\boldsymbol{r}^{\prime}\right) \nabla W \mathrm{~d} \boldsymbol{r}^{\prime}= & f(\boldsymbol{r}) \int_{\Omega} \nabla W \mathrm{~d} \boldsymbol{r}^{\prime}+\frac{\partial f(\boldsymbol{r})}{\partial x} \int_{\Omega}\left(x^{\prime}-x\right) \nabla W \mathrm{~d} \boldsymbol{r}^{\prime} \\
& +\frac{\partial f(\boldsymbol{r})}{\partial y} \int_{\Omega}\left(y^{\prime}-y\right) \nabla W \mathrm{~d} \boldsymbol{r}^{\prime}+O\left(h^{2}\right),
\end{aligned}
$$


Connecting Eq. (20) with the kernel approximation equation

$$
\langle\nabla f(\boldsymbol{r})\rangle=\int_{\Omega} f\left(\boldsymbol{r}^{\prime}\right) \nabla W \mathrm{~d} \boldsymbol{r}^{\prime}-f(\boldsymbol{r}) \int_{\Omega} \nabla W \mathrm{~d} \boldsymbol{r}^{\prime} .
$$

A new expression of the kernel gradient will be obtained

$$
\langle\nabla f(\boldsymbol{r})\rangle=\frac{\partial f(\boldsymbol{r})}{\partial x} \int_{\Omega}\left(x^{\prime}-x\right) \nabla W \mathrm{~d} \boldsymbol{r}^{\prime}+\frac{\partial f(\boldsymbol{r})}{\partial y} \int_{\Omega}\left(y^{\prime}-y\right) \nabla W \mathrm{~d} \boldsymbol{r}^{\prime}+O\left(h^{2}\right) .
$$

The particle approximation model can be written as:

$$
\left\langle\nabla f\left(\boldsymbol{r}_{i}\right)\right\rangle=\frac{\partial f\left(\boldsymbol{r}_{i}\right)}{\partial x_{i}} \sum_{j}\left(x_{j}-x_{i}\right) \nabla_{i} W_{i j} V_{j}+\frac{\partial f\left(\boldsymbol{r}_{i}\right)}{\partial y_{i}} \sum_{j}\left(y_{j}-y_{i}\right) \nabla_{i} W_{i j} V_{j} .
$$

Finally, the kernel gradient correction model is obtained

$$
\begin{aligned}
\nabla_{i}^{\text {new }} W_{i j} & =L\left(\boldsymbol{r}_{i}\right) \nabla_{i} W_{i j}, \\
L\left(\boldsymbol{r}_{i}\right) & =\left(\sum_{j}\left(\begin{array}{cc}
x_{j i} \frac{\partial W_{i j}}{\partial x_{i}} & y_{j i} \frac{\partial W_{i j}}{\partial x_{i}} \\
x_{j i} \frac{\partial W_{i j}}{\partial y_{i}} & y_{j i} \frac{\partial W_{i j}}{\partial y_{i}}
\end{array}\right) V_{j}\right)^{-1} .
\end{aligned}
$$

\subsection{Solid boundary treatment}

In the simulation, free-slip condition will be enforced over solid boundaries. To achieve the purpose, ghost particle method [Takeda et al. (1994); Morris et al. (1997)] is used. When the distance between the solid boundary particle and the fluid particle reaches some value, a ghost particle will be generated. The tangential velocities of the two particles are same, and the normal velocities are reverse.

$$
\left\{\begin{array}{ll}
x_{g}=2 x_{w}-x_{f} & y_{g}=2 y_{w}-y_{f} \\
v_{g, t}=v_{f, t} & v_{g, n}=-v_{f, n} \\
p_{g}=p_{f} & \rho_{g}=\rho_{f}
\end{array},\right.
$$

where $f$ is the fluid particle, $g$ is the ghost particle, and $w$ is corresponding wall particle. $v_{g, t}$ and $v_{f, t}$ are the tangential velocities of the ghost particle and the fluid particle and $v_{g, n}$ and $v_{f, n}$ are the normal velocities. The sketch is given in Fig. 1.

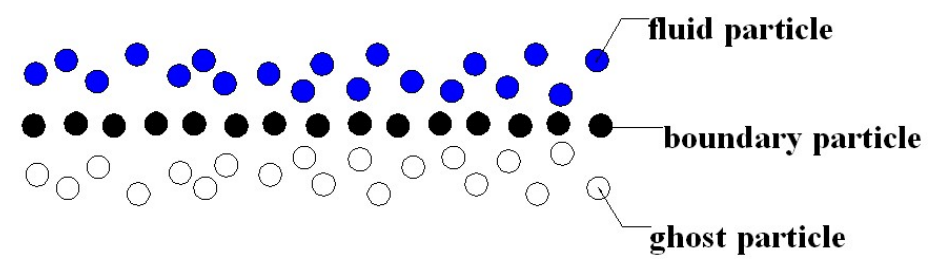

Fig. 1. The sketch of the fluid, boundary, and ghost particles. 


\section{RANS Turbulence Model}

In many case, the viscosity term is too dissipative and affects the shear in the fluid. When capturing the turbulence structure in SPH simulation, this problem is particularly important. Therefore, the RANS equations, obtained by time averaging the N-S equation, are employed in the simulation. First, new variables are obtained by time averaging,

$$
\bar{\phi}=\lim \frac{1}{t} \int_{t_{0}}^{t_{0}+t} \phi \mathrm{d} t .
$$

The transient volume $\phi$ in the turbulence is divided into two parts; the average volume $\bar{\phi}$ and the pulse volume $\phi^{\prime}, \phi=\bar{\phi}+\phi^{\prime}$. Using this method, the Reynolds average equations can be described as followed:

$$
\begin{aligned}
\frac{\mathrm{d} \rho}{\mathrm{d} t} & =-\rho \nabla \bullet \boldsymbol{V}, \\
\frac{\mathrm{d} V}{\mathrm{~d} t} & =-\frac{1}{\rho} \nabla P+\frac{\mu}{\rho} \nabla^{2} \boldsymbol{V}+\boldsymbol{g}+\frac{1}{\rho} \nabla(\rho \mathbf{R}),
\end{aligned}
$$

where $\boldsymbol{V}$ and $P$ are the new averaged volume of the original velocity and pressure and $R$ is the Reynolds strain tensor. In the Eq. (29), a new variable $R$ is introduced, and the equations are no longer closed. To resolve the problem, an eddy viscosity assumption is used [Lo and Shao (2002)].

$$
\begin{aligned}
R_{i j} & =2 \boldsymbol{\nu}_{t} S_{i j}-\frac{2}{3} k \delta_{i j}, \\
\boldsymbol{\nu}_{t} & =l^{2} \sqrt{2 S_{i j} S_{i j}}, \\
S_{i j} & =\frac{1}{2}\left(\frac{\partial \boldsymbol{V}_{j}}{\partial x_{i}}+\frac{\partial \boldsymbol{V}_{i}}{\partial x_{j}}\right),
\end{aligned}
$$

where $\boldsymbol{\nu}_{t}$ is the turbulence eddy viscosity, $S_{i j}$ is the average strain rate tensor, and $k$ is the kinetic energy, $l$ is the maxing length, $l=C_{s} h, c_{s}$ is the Smagorinsky constant, in this paper, $c_{s}=0.12$. Finally, the tensor term is represented by

$$
\frac{1}{\rho} \nabla(\rho \boldsymbol{R})=\sum_{j=1}^{N}\left(\frac{R_{i}}{\rho_{i}}+\frac{R_{j}}{\rho_{j}}\right) \nabla_{i} W_{i j} .
$$

\section{Numerical Examples}

In this section, the modified SPH method is used to simulate the dam-breaking problems. Although it is not a new attempt for SPH to model such problems, the purpose of the work is to validate the effects of the kernel gradient correction and the RANS turbulence model, which can lead to in-depth results and findings. In the simulation, two dam-break models are used; one has a gate controlling the discharge of water and the other not, as shown in Figs. 2 and 3. 


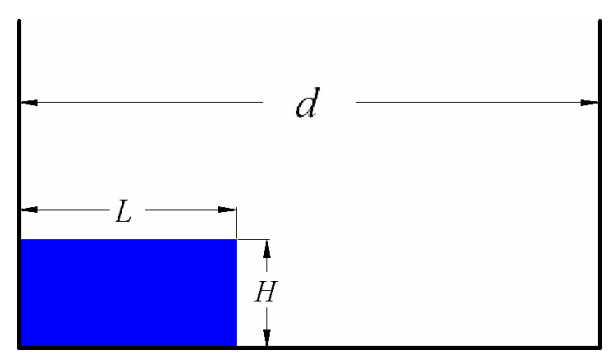

Fig. 2. The model of dam-break flow against a vertical wall.

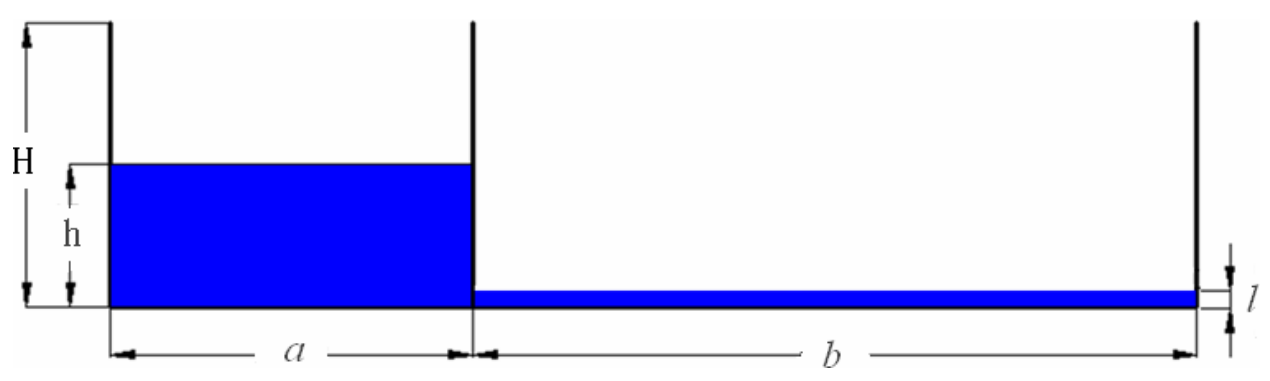

Fig. 3. The model of dam-break flow with a controlling gate.

\subsection{The effects of KGC-SPH model with density correction}

The model in Fig. 2 is used in this case. Here $L=2 \mathrm{H}, d=5.366 \mathrm{H}, H=0.6 \mathrm{~m}$. The time step is $0.00001 \mathrm{~s}$, the speed of sound is $40 \mathrm{~m} / \mathrm{s}$, and the coefficient of artificial viscosity is 0.08 . About 15,000 particles are used to represent the fluid. In the simulation, the ghost particle boundary is used.

At first, an initial pressure field is given basing on the height $\left(P_{i}=\rho g h_{i}\right)$, because in most conditions, the theoretical solutions of the initial pressure cannot be obtained Fig. 4 .

After several time steps, the pressure field will be similar with the theoretical values, as shown in Fig. 5, It also validates the adaptive nature of SPH.

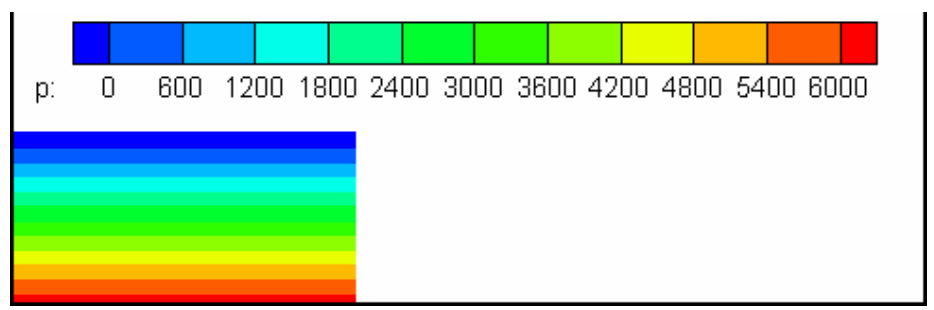

Fig. 4. The initial pressure field of the dam-break flow against a vertical wall. 


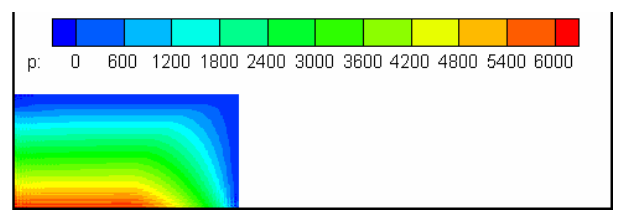

(a)

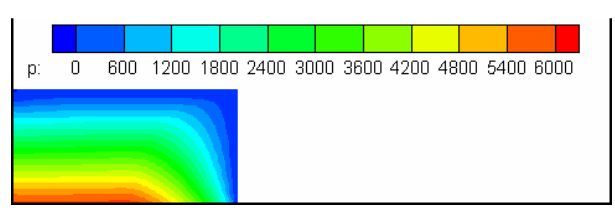

(b)

Fig. 5. The pressure field of the dam-break problem in $0.01 \mathrm{~s}$ : (a) without KGC and density correction and (b) with KGC and density correction.

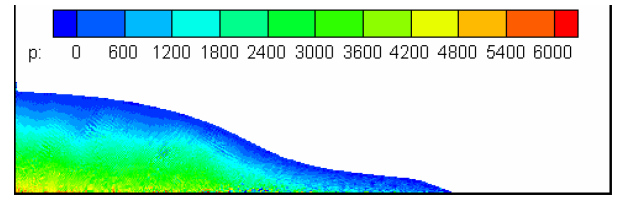

(a)

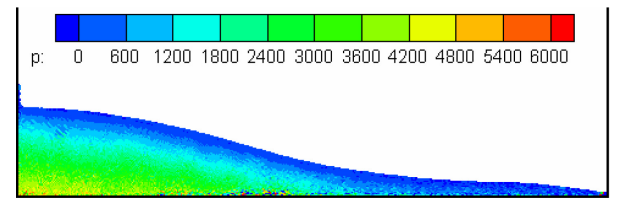

(b)

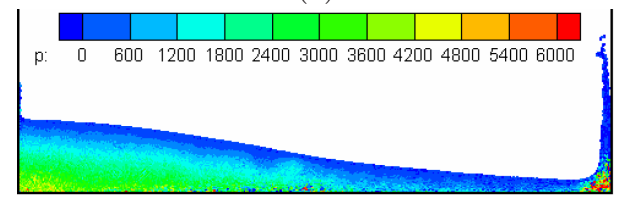

(c)

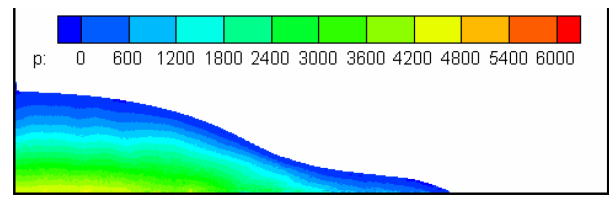

(d)

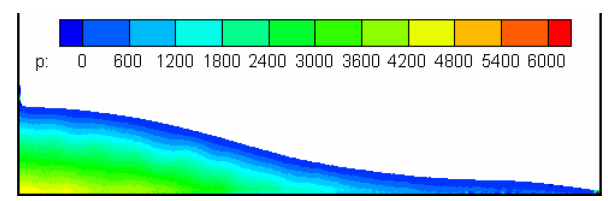

(e)

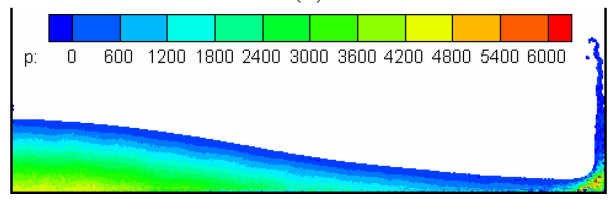

(f)

Fig. 6. The pressure field of the dam-break problem in $0.2,0.4$ and $0.8 \mathrm{~s}:(\mathrm{a}),(\mathrm{b})$, and (c) without KGC and density correction and (d), (e) and (f) with KGC and density correction.

To show the effect of the correction method, here, three flow forms in different time are given.

In Fig. 6, the left parts show the results from conventional SPH simulation and the right parts show the results from the improved SPH method, in which KGCmodel with density correction (renormalize the kernel function) is used. It is seen that the conventional SPH method can predict the pressure field to some extent; however, the pressure field is not smoothed, with some pressure oscillations. In the boundary, the pressure is also not accurate, and with the flowing of the fluid, the pressures of the water front become confused. It is difficult to make sure the explicit pressure values using conventional SPH method. In contrast, the improved $\mathrm{SPH}$ model with KGC and density correction produces much smoother pressure field. Using the kernel gradient correction and density correction, the consistency of the kernel function is restored and the accuracy of the kernel gradient is improved. 
Therefore, the pressure layers are clear, and little oscillations appear in the whole pressure field.

\subsection{The effects of RANS turbulence model with pressure correction}

To validate the roles of the RANS in describing the turbulence effects, a new case like the model in Fig. 3 is simulated; the difference is that no gate is used. Here, about 70000 particles are used, $\mathrm{H}=0.18 \mathrm{~m}, \mathrm{~h}=0.15 \mathrm{~m}, \mathrm{l}=0.018 \mathrm{~m}, " \mathrm{a}=0.3 \mathrm{~m} 8$ ", $\mathrm{b}=0.76 \mathrm{~m}$. Beside the RANS, KGC with MLS correction are also be used. The time step is $0.000005 \mathrm{~S}$, the speed of sound is $20 \mathrm{~m} / \mathrm{s}$, and the coefficient of artificial viscosity is 0.08 . At the same time, the Ghost particle boundary is used.

Similar with the last case, the initial pressure field is given based on the height; after several time steps, it will change like the sketch in Fig. 7.

To validate the accuracy of the simulation results, we compare the simulation with the experiment results.

In Figs. 8 and 9, it is shown that the flow patterns of the simulation are similar with the experiment results. In the simulation, the KGC and MLS corrections are used to smooth the pressure. In addition, the pressure also shows the effects of the correction. In the free surface, the pressure is stable; the KGC plays an important role. The layers of pressure are also obvious, and their transition is stable and suitable.

The two sketches both describe the turbulence effects successfully, especially in Fig. 9. The cavities are showed from their generations, developments to disappearance, and it is similar with the experiment. However, there is still a problem existed in the simulation. When the fluid begins to break, the matrix used in the kernel gradient correction may become singular, and this condition will stop the numerical simulation. Though the idea of variable smooth length can resolve the problem in some extent, it will added larger calculated amount. Therefore, a new corrected matrix will be tried to construct. In the simulation in this paper, in the areas involving singular matrix, the KGC model are not be used.

\section{Conclusions}

An improved SPH is introduced to simulate the classical dam-break problems. The results demonstrated that: (1) both conventional and improved SPH methods can

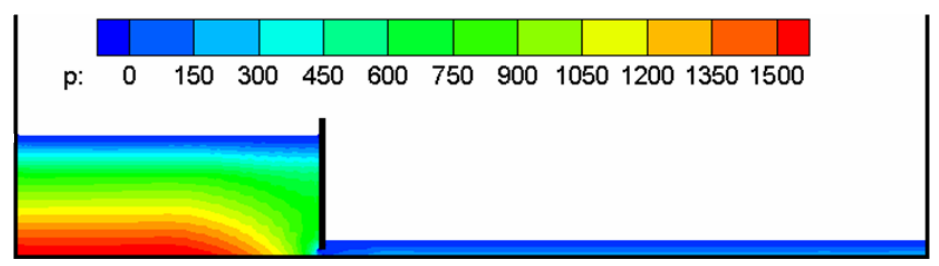

Fig. 7. The initial pressure field in $0.01 \mathrm{~S}$ of the dam-break problem with a gate. 

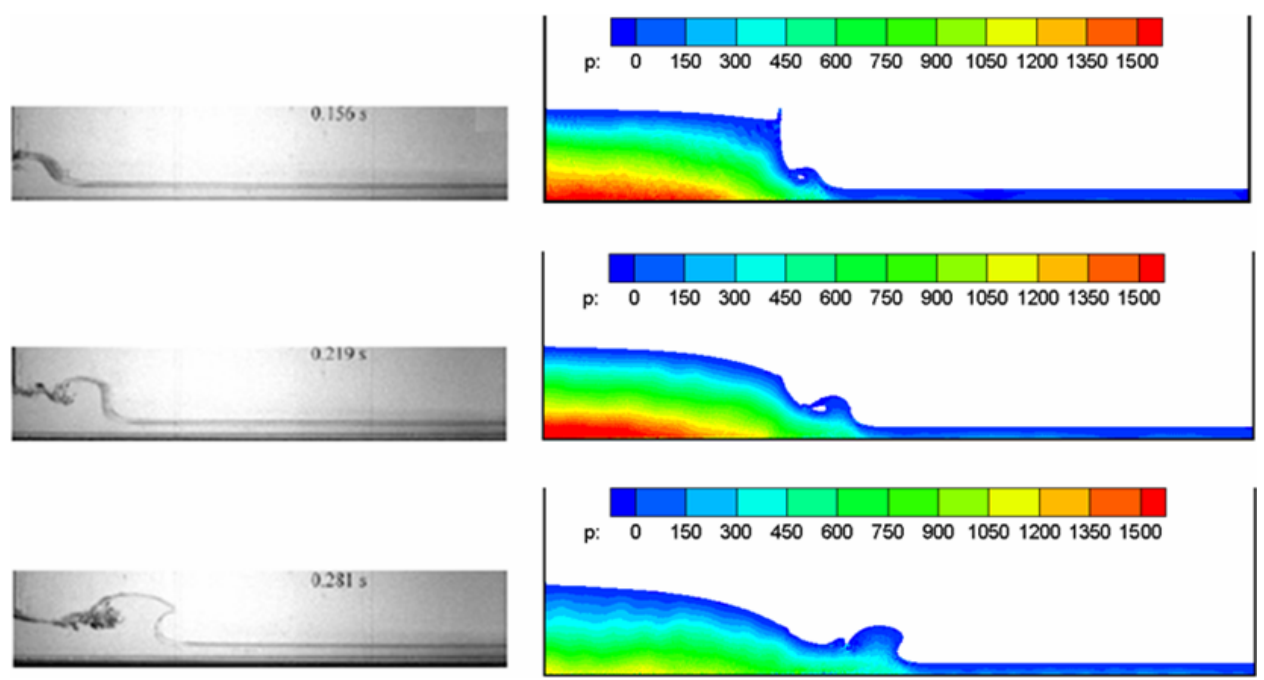

Fig. 8. The flow patterns of the dam-break problem with a gate in $0.156,0.219$, and $0.281 \mathrm{~s}$.
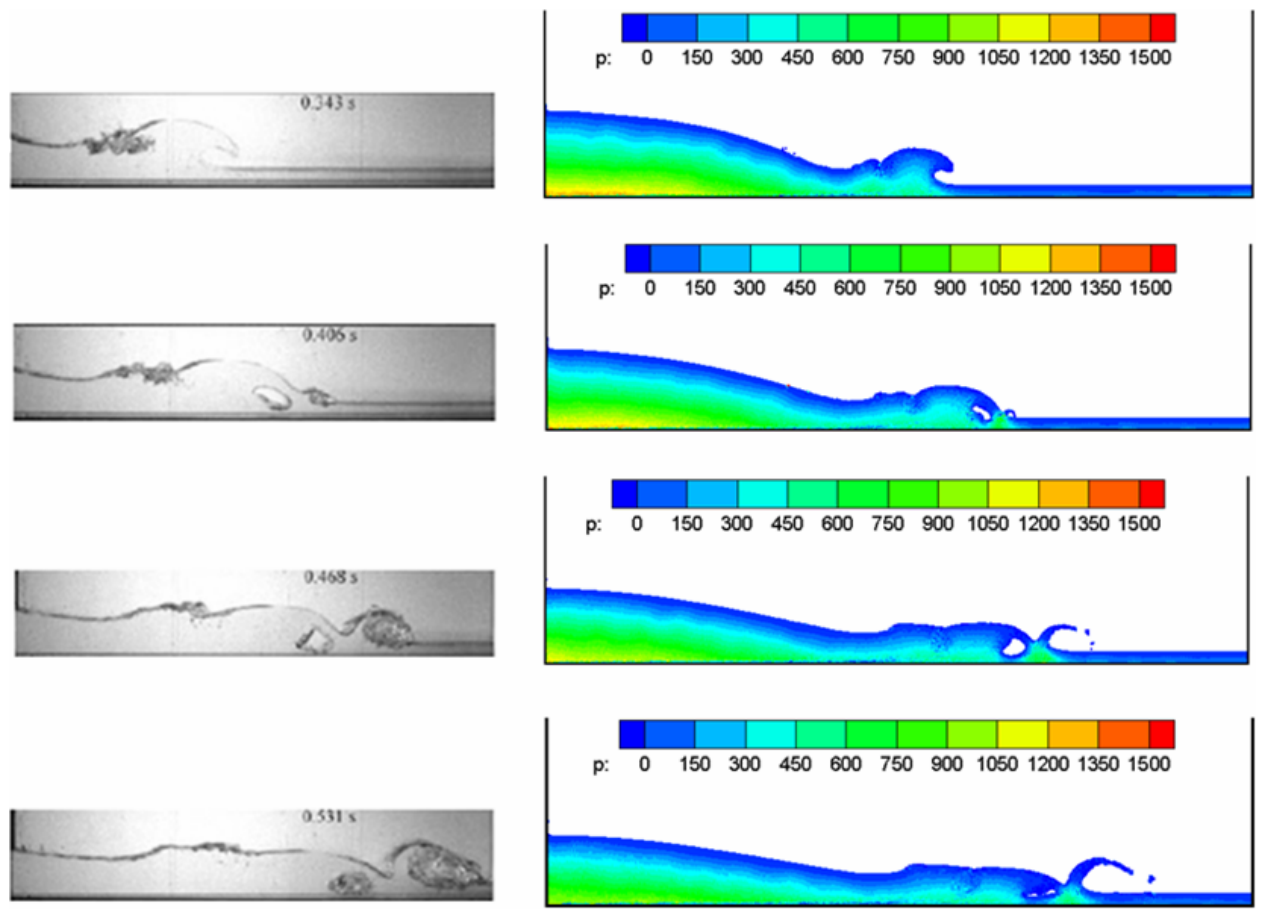

Fig. 9. The development of the cavities of the dam-break problem with a gate in $0.343,0.406,0.468$, and $0.531 \mathrm{~s}$. 
obtain acceptable results; they can provide a prediction of the pressure field in dam-break problems. (2) The KGC-model with density correction can remove the pressure oscillations and obtain more smoothed pressure field. (3) The use of RANS model can describe the inherent flow turbulence effectively, especially in the areas with flow cavities. In future, new work will be done, involving the treatments of matrix singularity problem and better method for complex solid boundaries.

\section{References}

Anderson, J. D. and Wendt, J. [1995] Computational Fluid Dynamics: The Basics with Applications, McGraw-Hill, New York.

Berendsen, H., Postma, J., van Gunsteren, W., DiNola, A., and Haak, J. [1984] Molecular dynamics with coupling to an external bath, J. Chem. Phy. 81, 3684.

Bonet, J. and Lok, T. [1999] Variational and momentum preservation aspects of smooth particle hydrodynamic formulations, Comput. Med. Appl. Mech. Eng. 180(1-2) 97-115.

Buchner, B. [2002] Green water on ship-type offshore structures, Ph.D Thesis, Delft University of Technology.

Catalano, P. and Amato, M. [2003] An evaluation of rans turbulence modelling for aerodynamic applications, Aeros. Sci. Tech. 7(7), 493-509.

Chen, C. J. and Jaw, S. [1998] Fundamentals of Turbulence Modeling, CRC, Press New York.

Dalrymple, R. A. and Rogers, B. D. [2006] Numerical modeling of water waves with the SPH method, Coast. Eng. 53(2-3), 141-147.

Faltinsen, O. M., Landrini, M. and Greco, [2004] Slamming in marine applications, J. Eng. Math. 48(3), 187-217.

Gingold, R. A. and Monaghan, J. J. [1977] Smoothed particle hydrodynamics - theory and application to non-spherical stars, Mon. Notic. Roy. Astron. Soc. 181, 375-389.

Groot, R. D. and Warren, P. B. [1997] Dissipative particle dynamics: bridging the gap between atomistic and mesoscopic simulation, J. Chem. Phys. 107(11), 4423.

Hirt, C. W. and Nichols, B. D. [1981] Volume of fluid (vof) method for the dynamics of free boundaries, J. Comput. Phys. 39(1), 201-225.

$\mathrm{Hu}, \mathrm{C}$. and Kashiwagi, M. [2004] A cip-based method for numerical simulations of violent free-surface flows, J. Mar. Sci. Technol. 9(4), 143-157.

Jánosi, M. I., Jan. D., Szabó, K. G., and Tél, T. [2004] Turbulent drag reduction in dambreak flows, Exp. Fluid. 37(2), 219-229.

Liu, G. R. and Liu, M. B. [2003] Smoothed Particle Hydrodynamics: A Meshfree Particle Method, World Scientific Pub. Co. Inc, Singapore.

Liu, M. B., Liu, G. R., and Zong, Z. [2008] An overview on smoothed particle hydrodynamics, Int. J. Comput. Meth. 5(1), 135-188.

Liu, M. B., Xie, W. P., and Liu, G. R. [2005] Modeling incompressible flows using a finite particle method, App. Math. Modelling. 29(12), 1252-1270.

Lo, Y. M. E. and Shao, S. D. [2002] Simulation of near-shore solitary wave mechanics by an incompressible sph method, Appl. Ocean. Res., 24(5), 275-286.

Lucy, L. B. [1977] A numerical approach to the testing of the fission hypothesis, Astron. J. 82, 1013-1024.

Mei, C. C. [1989] The Applied Dynamics of Ocean Surface Waves, Advanced Series on Ocean Engineering, World Scientific, Singapore. 
Moin, P. and Mahesh, K. [1998] Direct numerical simulation: a tool in turbulence research, Annu. Rev. Fluid. Mech. 30(1), 539-578.

Monaghan, J. J. [1985] Particle methods for hydrodynamics, Comput. Phys. Rep. 3, 71124.

Monaghan, J. J. [1994] Simulating free surface flows with SPH, J. Comput. Phys. 110, 399-399.

Monaghan, J. J. [2005] Smoothed particle hydrodynamics, Rep. Prog. Phys. 68, 1703-1759.

Morris, J. P., Fox, P. J., and Zhu, Y. [1997] Modeling low Reynolds number incompressible flows using SPH, J. Comput. Phys. 136(1), 214-226.

Moser, R. D., Kim, J., and Mansour, N. N. [1999] Direct numerical simulation of turbulent channel flow up to re $=590$, Phys. Fluid. 11, 943.

Peregrine, D. H. [2003] Water-wave impact on walls, Ann. Rev. Fluid. Mech. 35, 23-43.

Sethian, J. [2003] Level set methods and fast marching methods, J. Comput. Inf. Technol. 11(1), 1-2.

Takeda, H., Miyama, S. M., and Sekiya, M. [1994] Numerical simulation of viscous flow by smoothed particle hydrodynamics, Prog. Theor. Phys. 92(5), 939-960. 